\title{
2-GROUPS WITH EVERY AUTOMORPHISM CENTRAL
}

\author{
S. P. GLASBY \\ (Received 12 September 1984) \\ Communicated by D. E. Taylor
}

\begin{abstract}
An infinite family of 2 -groups is produced. These groups have no direct factors and have a non-abelian automorphism group in which all automorphisms are central.

1980 Mathematics subject classification (Amer. Math. Soc.): 20 F 28, 20 D 45.

The automorphisms of a group $G$ which induce the identity on $G / Z(G)$ are called central. This paper investigates groups which admit only central automorphisms. Various authors ([2], [5], [7], [9]) considered non-abelian $p$-groups with abelian automorphism groups in which necessarily every automorphism is central. Curran [1] and Malone [6] constructed non-abelian $p$-groups with non-abelian automorphism groups in which every automorphism is central. The groups constructed by Curran and Malone, however, all had direct factors. Malone [6] wondered if this condition were necessary. The groups $G_{n}(n \geqslant 4)$ described below show that direct factors are not necessary.

Define $G_{n}$ to be the group

$$
\begin{aligned}
\left\langle x_{1}, \ldots, x_{n}\right| x_{i}^{2^{i}}=1,1 \leqslant i \leqslant n,\left[x_{i}, x_{i+1}\right]= & x_{i+1}^{2^{i}}, 1 \leqslant i<n, \\
& \left.=\left[x_{i}, x_{j}\right]=1,1<i+1<j \leqslant n\right\rangle .
\end{aligned}
$$
\end{abstract}

Then $G_{n}$ has order $2^{n(n+1) / 2}$. The group $G_{3}$ was first considered by Miller [7] and is group 99 of the Hall and Senior tables [4].

The author gratefully acknowledges the support of a Commonwealth Postgraduate Research Award. (C) 1986 Australian Mathematical Society $0263-6115 / 86 \$ A 2.00+0.00$ 
THEOREM. For $n \geqslant 3, G_{n}$ has no direct factors and only central automorphisms. The automorphism group of $G_{n}$ has order $2^{p(n)}$, where $p(n)=(n-1)\left(2 n^{2}-n+\right.$ 6) $/ 6$, and is non-abelian for $n \geqslant 4$.

Proof. The centre and derived subgroups of $G_{n}$ are $\left\langle x_{2}^{2}, x_{3}^{2}, \ldots, x_{n}^{2}\right\rangle$ and $\left\langle x_{2}^{2}, x_{3}^{4}, \ldots, x_{n}^{2^{n-1}}\right\rangle$, respectively. Let $x\left(i_{1}, \ldots, i_{n}\right)$ denote $x_{1}^{i_{1}} \cdots x_{n}^{i_{n}}$. Then every element of $G_{n}$ may be uniquely represented as $x\left(i_{1}, \ldots, i_{n}\right)$ with $0 \leqslant i_{k}<2^{k}$. Commutator collection, together with the fact that $G_{n}$ has class 2, yields the equation

$$
x\left(i_{1}, \ldots, i_{n}\right)^{2}=x\left(0,2 i_{2}\left(1+i_{1}\right), 2 i_{3}\left(1+2 i_{2}\right), \ldots, 2 i_{n}\left(1+2^{n-2} i_{n-1}\right)\right) .
$$

Thus the square of every element is central. Since the centre has exponent $2^{n-1}$, $G_{n}$ has exponent $2^{n}$. All elements of maximal order must have $i_{n}$ odd.

Suppose $G_{n}$ is the direct product $A \times B$, where $A$ has exponent $2^{n}$. If $y=x_{n}^{2^{n-1}}$, then the $2^{n-1}$ th power of every element of order $2^{n}$ is $y$. Thus $y$ is an element of $A$, and no element of $B$ has order $2^{n}$. Hence every element of order $2^{n}$ is contained in $A$, and so $A$ contains $x_{1} x_{n}, \ldots, x_{n-1} x_{n}, x_{n}$ and must therefore equal $G_{n}$.

If $i_{k, j}$ are integers, then the map taking $x_{1}$ to $x\left(1,2 i_{1,2}, 4 i_{1,3}, \ldots, 2^{n-1} i_{1, n}\right)$ and $x_{k}$ to $x\left(2 i_{k, 1}, \ldots, 2 i_{k, k-1}, 2 i_{k, k}+1,2^{2} i_{k, k+1}, \ldots, 2^{n-k+1} i_{k, n}\right), 1<k \leqslant n$, defines a homomorphism of $G_{n}$ which is the identity on $G_{n} / Z\left(G_{n}\right)$. Indeed, because the centre of $G_{n}$ equals the Frattini subgroup, these homomorphisms are automorphisms. There are $2^{p(n)}$ distinct automorphisms of the above form.

As $G_{n}$ has no abelian direct factors, [8, Theorem 1] may be used to show that $G_{n}$ has $2^{p(n)}$ central automorphisms. We shall prove by induction that all automorphisms of $G_{n}$ are central for $n \geqslant 3$, and thus all have the above form. The case $n=3$ is easy because $p(3)=7$ and the Miller group, $G_{3}$, has $2^{7}$ automorphisms (see $[4,7])$.

Suppose $n>3$. The above remarks show that $y$ is fixed by all automorphisms of $G_{n}$. There is an isomorphism ' from $G_{n} /\langle y\rangle$ to $G_{n-1} \times Z$ such that the elements $x_{1}^{\prime}, \ldots, x_{n-1}^{\prime}$ generate $G_{n-1}$, and such that the element $x_{n}^{\prime}$ of order $2^{n-1}$ generates $Z$. The homomorphism ${ }^{*}$ from $\operatorname{Aut}\left(G_{n}\right)$ to $\operatorname{Aut}\left(G_{n-1} \times Z\right)$ and the inductive hypothesis give information about $\operatorname{Aut}\left(G_{n}\right)$.

Let $\iota$ be the inclusion map from $G_{n-1}$ to $G_{n-1} \times Z, \pi$ the projection map from $G_{n-1} \times Z$ to $G_{n-1}$, and $\phi$ an automorphism of $G_{n}$. Then, arguing as in Malone [6], we see that $\iota \phi^{*} \pi$ is an automorphism of $G_{n-1}$. So any automorphism of $G_{n}$ takes $x_{1}$ to $x\left(1,2 i_{1,2}, \ldots, 2^{n-2} i_{1, n-1}, j_{1, n}\right)$ and $x_{k}$ to $x\left(2 i_{k, 1}, \ldots, 2 i_{k, k-1}, 2 i_{k, k}+\right.$ $\left.1,2^{2} i_{k, k+1}, \ldots, 2^{n-k_{i}} i_{k, n-1}, j_{k, n}\right), 1<k<n$. But $x_{k}$ is mapped to an element of order $2^{k}$, so $2^{n-k}$ divides $j_{k, n}$ for $1 \leqslant k<n$. Since $x_{n}^{\prime}$ is central and $x_{n}$ is not, $x_{n}$ is mapped to $x\left(2 i_{n, 1}, \ldots, 2 i_{n, n-1}, 2 i_{n, n}+1\right)$. Thus all automorphisms of $G_{n}$ are central, which completes the inductive proof. 
Finally, $\operatorname{Aut}\left(G_{n}\right)$ is non-abelian for $n \geqslant 4$. Let $\phi$ be the automorphism which maps $x_{4}$ to $x_{3}^{2} x_{4}$ and which fixes the remaining generators. Let $\psi$ map $x_{3}$ to $x_{3} x_{4}^{4}$ and fix the other generators. Then $\left(x_{4}\right) \phi \psi=x_{3}^{2} x_{4}^{9}$ and $\left(x_{4}\right) \psi \phi=x_{3}^{2} x_{4}$. Thus $\phi$ and $\psi$ do not commute, and so the proof of the theorem is complete.

FURTHER EXAMPLES. We exhibit some further examples of 2-groups with all automorphisms central. Let $H_{n}$ and $K_{n}$ denote the groups

$$
\begin{gathered}
H_{n}=\left\langle x_{1}, \ldots, x_{n} \mid x_{i}^{4}=1,1 \leqslant i \leqslant n,\left[x_{i}, x_{n}\right]=x_{i+1}^{2}, 1 \leqslant i<n\right\rangle, \text { and } \\
K_{n}=\left\langle x_{0}, \ldots, x_{n}\right| x_{0}^{2}=x_{i}^{4}=x_{n}^{2}=1,1 \leqslant i<n,\left[x_{0}, x_{i}\right]=x_{i+1}^{2}, \\
\left.1 \leqslant i<n-1,\left[x_{0}, x_{n-1}\right]=x_{\bar{n}}\right\rangle,
\end{gathered}
$$

where the commutators $\left[x_{i}, x_{j}\right](1 \leqslant i<j \leqslant n)$ not shown above are trivial. A proof of the statements made below appears in [3], a copy of which may be obtained from the author. The groups $H_{n}$ and $K_{n}$ have order $2^{2 n}$, and their automorphism groups are elementary abelian of order $2^{n^{2}}$. Furthermore, $H_{n}$ and $K_{n}$ are not isomorphic either to themselves or to any of Jonah and Konvisser's groups [5].

The groups $G_{n}$, like the groups $H_{n}$ and $K_{n}$, may be generalized to give different groups whose automorphisms are all central. Let $G\left(l_{1}, \ldots, l_{n}\right)$ denote the group

$$
\begin{aligned}
\left\langle x_{1}, \ldots, x_{n}\right| x_{i}^{m_{i}}=1,1 \leqslant i \leqslant n,\left[x_{i-1}, x_{i}\right]=x_{i}^{m_{i} / 2}, 1<i \leqslant n, & \\
{\left[x_{i}, x_{j}\right] } & =1,1<i+1<j \leqslant n\rangle,
\end{aligned}
$$

where $n \geqslant 3, m_{i}=2^{l_{i}}$ and $0<l_{1}<\cdots<l_{n}$. Then every automorphism of $G\left(l_{1}, \ldots, l_{n}\right)$ is central if and only if $l_{1}=1$ and $l_{2}=2$. The groups $G(1,2, l)$ are isomorphic to those considered by Struik [9], and the group $G(1, \ldots, n)$ is isomorphic to $G_{n}$. Finally, $G\left(1,2, \ldots, l_{n}\right)$ has no direct factors and, when $n>3$, has a non-abelian automorphism group of order $2^{p}$, where $p=2(n-1)+$ $\sum_{k=3}^{n}\left[\left(l_{1}+l_{2}+\cdots+l_{k}\right)-k+(n-k)(k-1)\right]$.

\section{References}

[1] M. J. Curran, 'A non-abelian automorphism group with all automorphisms central', Bull. A ustral. Math. Soc. 26 (1982), 393-397.

[2] R. Faudree, 'Groups in which each element commutes with its endomorphic images', Proc. Amer. Math. Soc. 27 (1971), 236-240.

[3] S. P. Glasby, 2-groups with every automorphism central (preprint, The University of Sydney, 1984).

[4] M. Hall, Jr. and J. Senior, The groups of order $2^{n}(n \leqslant 6)$ (Macmillan, New York; Collier-Macmillan, London, 1964). 
[5] D. Jonah and M. Konvisser, 'Some non-abelian p-groups with abelian automorphism groups', Arch Math. (Basel) 26 (1975), 131-133.

[6] J. J. Malone, ' $p$-groups with non-abelian automorphism groups and all automorphisms central', Bull. Austral. Math. Soc. 29 (1984), 35-37.

[7] G. A. Miller, 'A non-abelian group whose group of automorphisms is abelian', Messenger Math . $43(1913 / 1914), 124-125$.

[8] P. R. Saunders, 'The central automorphisms of a finite group', J. London Math. Soc. 44 (1969), 225-228.

[9] R. R. Struik, 'Some non-abelian 2-groups with abelian automorphism groups', Arch. Math. (Basel) 39 (1982), 299-302.

\section{Department of Pure Mathematics}

The University of Sydney

New South Wales 2006

Australia 